\title{
STRUCTURAL, MAGNETIC, AND MAGNETO-OPTIC PROPERTIES OF MnBi(Al, Pt) THIN FILMS: THERE IS STILL MORE TO LEARN
}

\author{
P. Fumagalli, U. Rüdiger, P. Dworak, B. Holländer, ${ }^{\ddagger}$ U. Nowak, ${ }^{*}$ and G. Güntherodt \\ 2. Physikalisches Institut, Rheinisch-Westfälische Technische Hochschule Aachen, D-52056 Aachen, Germany \\ ${ }^{\ddagger}$ Institut für Schicht- und Ionentechnik (ISI), Forschungszentrum Jülich, D-52425 Jülich, Germany \\ * Theoretische Tieftemperaturphysik, Gerhard-Mercator-Universität Duisburg, D-47048 Duisburg, Germany
}

\begin{abstract}
MnBi thin films with Al and Pt interlayers have been prepared by a sequential technique. Structural, magnetic, and magneto-optic properties at room temperature are discussed. We find that surface roughness intensifies with increasing film thickness due to the growth condition of $\mathrm{Mn}$ on Bi. Surface roughness is efficiently reduced by adding a thin Al or Pt interlayer. The coercivity strongly depends on adding interlayers or a protective overlayer. If the additional layer forms a rigid barrier, the $\mathrm{MnBi}$ formation is restricted perpendicular to the film surface, leading to a pronounced lateral growth and lower coercive fields. This could be related to an increase of $2.3 \%$ in volume between a stoichiometric $\mathrm{Bi} / \mathrm{Mn}$ bilayer and the $\mathrm{MnBi}$ compound. The addition of $\mathrm{Al}$ interlayers does not reduce the Kerr effect considerably while a Pt interlayer reduces magnetization and, consequently, the Kerr effect.
\end{abstract}

KEYWORDS: MnBi, MAGNETO-OPTICS, KERR EFFECT, COERCIVITY, SURFACE ROUGHNESS, INTERLAYERS

\section{INTRODUCTION}

$\mathrm{MnBi}$ is one of the most intensively studied ferromagnetic materials and, yet, there are still open questions regarding its suitability as a magneto-optic recording media. $\mathrm{MnBi}$ shows an extraordinary large magneto-optic Kerr rotation of almost $2^{\circ}$ at room temperature (as measured through a glass substrate) over a wide photon energy range [1]. Therefore, $\mathrm{MnBi}$ is an interesting candidate for magneto-optic storage applications in the blue spectral range. The hexagonal NiAs structure provides the necessary perpendicular magnetic anisotropy. However, there are some major complications which made an application impossible up to now. (i) A structural phase transition takes place at 340$355^{\circ} \mathrm{C}$ from a ferromagnetic low-temperature phase to a paramagnetic high-temperature phase [2]. (ii) The coercive field, $H_{\mathrm{c}}$, increases with temperature showing a maximum at $280^{\circ} \mathrm{C}$ which is unfavorable for thermomagnetic writing [3]. (iii) Thin films deposited by the standard $\mathrm{Bi} / \mathrm{Mn}$-layering technique show a large grain. size which increases noise in magneto-optic recording. To our knowledge, nobody has yet succeeded to grow MnBi by co-evaporation which would yield a better control over structural properties.

We started our investigation by reviewing the standard deposition technique of $\mathrm{MnBi}$ films and by verifying the magnetic and magneto-optic properties [4]. In contrast to previous work, the $\mathrm{MnBi}$ films are not covered with a protective $\mathrm{SiO}_{x}$ layer in order to be able to use surfaceanalyzing methods like scanning electron microscopy (SEM) and atomic force microscopy (AFM). The influence of $\mathrm{Al}$ and Pt additions on structural, magnetic, and magneto-optic properties of $\mathrm{MnBi}$ thin films is discussed in this paper. First results on $\mathrm{Al}$ additions have been published before [5].

\section{EXPERJMENTAL}

The $\mathrm{MnBi}$ thin films are deposited as subsequent $\mathrm{Bi} / \mathrm{Mn}$ bilayers onto fused $\mathrm{SiO}_{2}$ and glass substrates at ambient temperatures in a vacuum of $10^{-6}$ mbar. $\mathrm{Al}$ or $\mathrm{Pt}$ is added by placing an interlayer between subsequent $\mathrm{Bi} / \mathrm{Mn}$ bilayers. A typical layering sequence is shown in Fig. 1. The thickness of the $\mathrm{Bi}$ and $\mathrm{Mn}$ layers is fixed at 18 and $12 \mathrm{~nm}$, respectively, to obtain after annealing the optimum stoichiometry. The deposition rate of $0.04 \mathrm{~nm} / \mathrm{s}$ for $B i$ is such that a $c$-axis texture of the Bi layers is achieved which acts as a template for the $\mathrm{MnBi}$ formation. The deposition rate for $\mathrm{Mn}$ and $\mathrm{Al}$ is $0.2 \mathrm{~nm} / \mathrm{s}$ and for Pt $0.01 \mathrm{~nm} / \mathrm{s}$. All films containing $\mathrm{Al}$ and $\mathrm{Pt}$ layers were annealed for one hour at temperatures between $300-380^{\circ} \mathrm{C}$ yielding films with $c$-axis orientation [5].

Magnetization measurements were performed on a commercial SQUID magnetometer. The polar Kerr spectra of all samples were measured through the substrate because of surface oxidation and poor reflectivity due to surface roughness while the Kerr hysteresis loops were obtained from both, the film and substrate side. The complex polar Kerr effect was measured in a fully-automated, high-resolution Kerr spectrometer over a wide photon energy range of 1.1$5.2 \mathrm{eV}$ in magnetic fields up to $2.77 \mathrm{~T}$ at room temperature. For each data point, the difference of the rotations of the sample and a magneto-optically inactive reference, usually an aluminum mirror, is calculated in order to account for any rotation of the substrate and optical components in the stray field of the magnet. In addition, all measurements are taken in both, positive and negative magnetic fields in order to eliminate any rotations of nonmagnetic origin, as e. g., stressinduced birefringence of the films. The reflectivity was derived directly from the Kerr measurements relative to the $\mathrm{Al}$ reference mirror and is not corrected for the substrate.

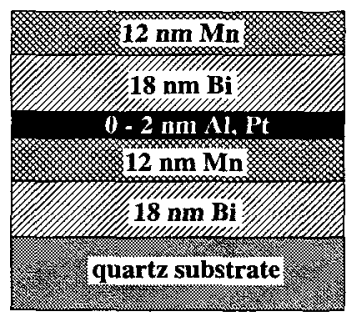

FIG. 1. Typical layering sequence of the $\mathrm{MnBi}(\mathrm{Al}, \mathrm{Pt})$ thin films. The thickness of the $\mathrm{Bi}$ and $\mathrm{Mn}$ layers is chosen to yield the optimum MnBi stoichiometry after annealing. 


\section{STRUCTURAL PROPERTIES}

First, the influence of $\mathrm{Al}$ and $\mathrm{Pt}$ addition on the structural properties of $\mathrm{MnBi}$ thin films is discussed. The addition of $\mathrm{Al}$ and $\mathrm{Pt}$ does not have much effect on the preferred $c$-axis orientation of the $\mathrm{MnBi}(\mathrm{Al}, \mathrm{Pt})$ thin films as is evident from $x$-ray diffraction analysis shown in Fig. 2. For the as-grown films (Fig. 2(a)), $\mathrm{Bi}(00 l)$ reflections are present indicating strong $c$-axis orientation. A few spurious features due to the sample holder are labeled with an asterisk. After annealing, the $\mathrm{MnBi}$ (Fig. 2(b)) as well as the films containing Al and Pt layers (Figs. 2(c) and (d), respectively) show strong $\mathrm{MnBi}(00 l)$ reflections indicating the hexagonal NiAs-type structure of $\mathrm{MnBi}$. The small shifts observed in $2 \Theta$ are due to a misalignment of the samples when mounting onto the $\mathrm{x}$-ray sample holder. Note that there is no evidence for a second phase, like $\mathrm{MnAl}, \mathrm{MnPt}$ or the high-temperature $\mathrm{Mn}_{1.08} \mathrm{Bi}$ phase, as has been found by other groups $[6,7]$. There is, however, a small amount of residual $\mathrm{Bi}$ in the annealed MnBi (Fig. 2(b)) and the MnBiPt film (Fig. 2(d)).

As the addition of $\mathrm{Al}$ or $\mathrm{Pt}$ neither reduces $c$-axis orientation nor changes the MnBi crystal lattice constant, the question arises what happens to the interlayers at all. In order to elucidate this question, Rutherford backscattering (RBS) experiments were performed using $1.4 \mathrm{MeV} \mathrm{He}{ }^{+}$ions and a scattering angle of $170^{\circ}[8]$. A comparison between the RBS spectra before (dashed line) and after (solid line) annealing of a $\mathrm{Bi} / \mathrm{Mn} / \mathrm{Al}(2 \mathrm{~nm}) / \mathrm{Bi} / \mathrm{Mn}$ and a $\mathrm{Bi} / \mathrm{Mn} / \mathrm{Pt}(2 \mathrm{~nm}) / \mathrm{Bi} / \mathrm{Mn}$ sample is displayed in Fig. 3. The surface-backscattering energies of the components are marked by vertical lines. The

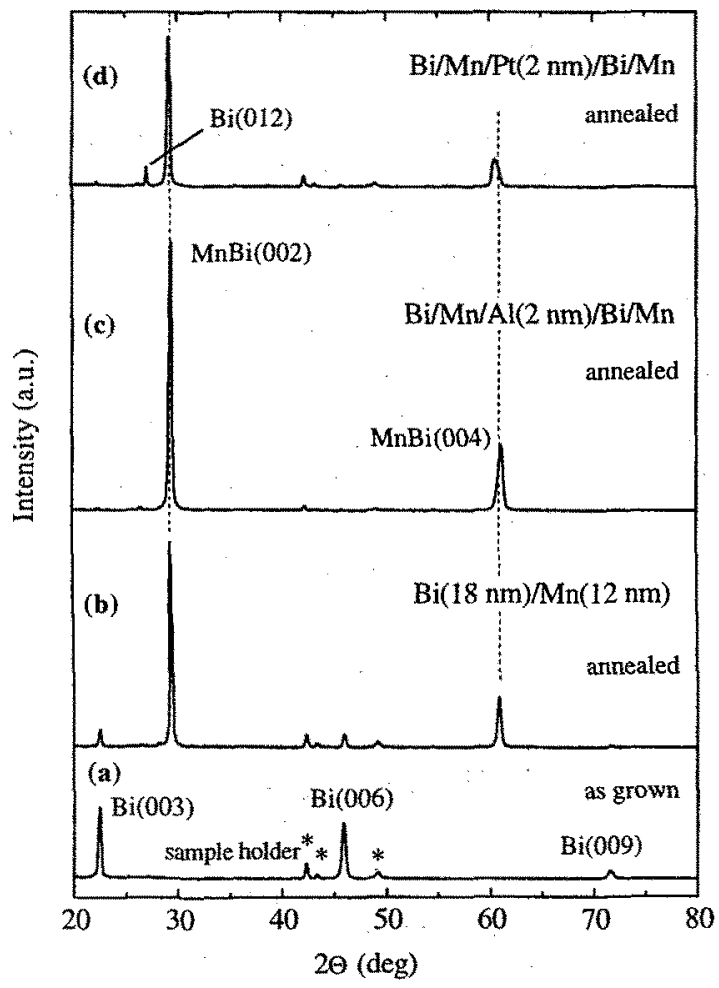

FIG. 2. X-ray diffraction spectra of $\mathrm{MnBi}(\mathrm{Al}, \mathrm{Pt})$ thin films before and after annealing.

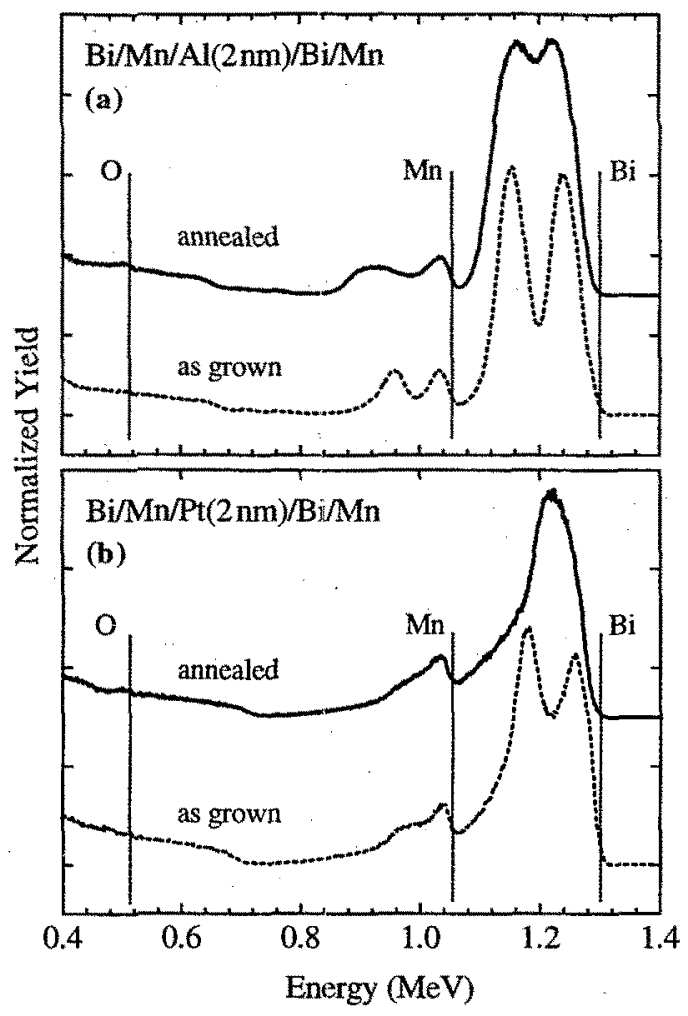

FIG. 3. Rutherford-backscattering spectra before (dotted line) and after (solid line) annealing of (a) a $\mathrm{Bi} / \mathrm{Mn} / \mathrm{Al}(2 \mathrm{~nm}) / \mathrm{Bi} / \mathrm{Mn}$ and (b) a $\mathrm{Bi} / \mathrm{Mn} / \mathrm{Pt}(2 \mathrm{~nm}) / \mathrm{Bi} / \mathrm{Mn}$ sequence. The vertical lines mark the surface-backscattering energy of the constituents.

offset to lower energies is indicative of the location of a component inside the film relative to the surface. Typically, heavy elements, like $\mathrm{Bi}$, have less energy loss than light elements, like $\mathrm{Mn}$ or $\mathrm{O}$. Focusing on the dominant $\mathrm{Mn}$ and $\mathrm{Bi}$ features, a distinct separation into two layers, represented by a double peak in the RBS spectra, is recognized in both systems in the as-grown state. This simply represents the layering sequence of the deposition (see Fig. 1).

After annealing, the separation is preserved to a great extent in the Al-doped system while the Pt-doped system reveals a complete interdiffusion of the two $\mathrm{Bi} / \mathrm{Mn}$ bilayers. A small amount of oxygen is present directly at the surface increasing after annealing by a factor of about 2.5 [8]. It most certainly forms with $\mathrm{Mn}$ a thin $\mathrm{Mn}_{3} \mathrm{O}_{4}$ surface layer. This is corroborated by the asymmetry of the $\mathrm{Mn}$ double peaks, especially in the $\mathrm{Bi} / \mathrm{Mn} / \mathrm{Pt} / \mathrm{Bi} / \mathrm{Mn}$ sample, implying a higher Mn density at the surface. No anisotropy is observed, however, in the $\mathrm{Bi} / \mathrm{Mn} / \mathrm{Al} / \mathrm{Bi} / \mathrm{Mn}$ sample before annealing. This could indicate that the Al interlayer getters some of the oxygen during deposition thus forming $\mathrm{Al}_{2} \mathrm{O}_{3}$, which is a very firm oxide. In addition, this would explain why the two $\mathrm{Bi} / \mathrm{Mn}$ bilayers are still separated after annealing. $\mathrm{Pt}$, being a noble metal, will not form an oxide and will hence be a minor diffusion barrier to the $\mathrm{MnBi}$ formation across the interlayer. As will be discussed in the next sections, the different structural characteristics of $\mathrm{Al}$ and $\mathrm{Pt}$ interlayers have a strong effect on the magnetic and magneto-optic properties. 


\section{MAGNETIC PROPERTIES}

We will briefly review the magnetic properties of pure $\mathrm{MnBi}$ films as a function of thickness [4]. The SEM pictures of a single $\mathrm{Bi} / \mathrm{Mn}$ bilayer in comparison to a $\mathrm{Bi} / \mathrm{Mn} / \mathrm{Bi} / \mathrm{Mn}$ double bilayer is shown in Fig. 4. By enlarging the film thickness $d_{\mathrm{MnBi}}$, i.e. the number of $\mathrm{Bi} / \mathrm{Mn}$ bilayers, surface roughness increases strongly during deposition and does not change after annealing. An AFM [9] study indicates that the surface roughness is generated during growth of $\mathrm{Mn}$ onto $\mathrm{Bi}$.

The magnetic properties of annealed films are changing as well. The polar Kerr hysteresis loops, measured from the film side, show a strong decrease of the coercive field, $H_{\mathrm{c}}$, from $1.25 \mathrm{~T}$ in a single $\mathrm{Bi} / \mathrm{Mn}$ bilayer $\left(d_{\mathrm{MnBi}}=41 \mathrm{~nm}\right)$ to $0.4 \mathrm{~T}$ in a $(\mathrm{Bi} / \mathrm{Mn})_{3}$ sequence $\left(d_{\mathrm{MnBi}}=90 \mathrm{~nm}\right)$, as plotted in Fig. 5. The reduction in $H_{\mathrm{c}}$ with increasing thickness can be understood qualitatively as an increase in $\mathrm{MnBi}$ grain size on the basis of a simple model which assumes spherical, singledomain particles [10]. From the SEM pictures, we can estimate a grain size of $200-300 \mathrm{~nm}$ in thick MnBi films. The high coercive fields are in contrast to earlier work $[3,11]$, where for a similar film thickness values of $H_{\mathrm{c}} \leq 0.5 \mathrm{~T}$ were measured. As will be discussed later, this is due to the altered growth condition when depositing a protective $\mathrm{SiO}_{x}$ layer.

Besides the decrease of $H_{c}$, an additional change in the characteristics of the magnetization reversal is observed. While a single $\mathrm{Mn} / \mathrm{Bi}$ bilayer is reversing smoothly, as revealed in a symmetric ' $S$ '-shaped slope of the inner loop (see Fig. 5(a)), a thick MnBi film exhibits a sudden onset of magnetization reversal before leveling off very slowly (see Fig. 5(b)).

In order to understand the magnetization reversal from a theoretical point of view, hysteresis loops were simulated by a Monte-Carlo method using a micromagnetic model which
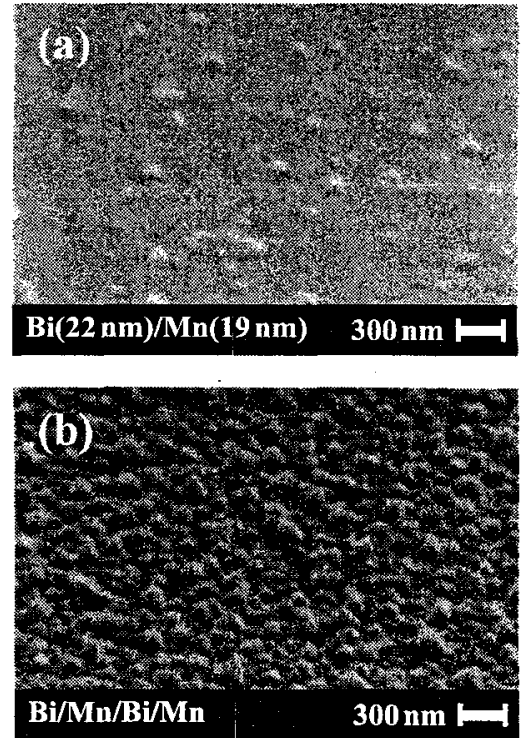

FIG. 4. SEM picture of (a) a $\mathrm{Bi}(22 \mathrm{~nm}) / \mathrm{Mn}(19 \mathrm{~nm})$ bilayer and (b) a $\mathrm{Bi} / \mathrm{Mn} / \mathrm{Bi} / \mathrm{Mn}$ double bilayer with a $\mathrm{Bi}$ and $\mathrm{Mn}$ layer thickness of 18 and $12 \mathrm{~nm}$, respectively. Both pictures are taken before annealing. The characteristics do not change after annealing.

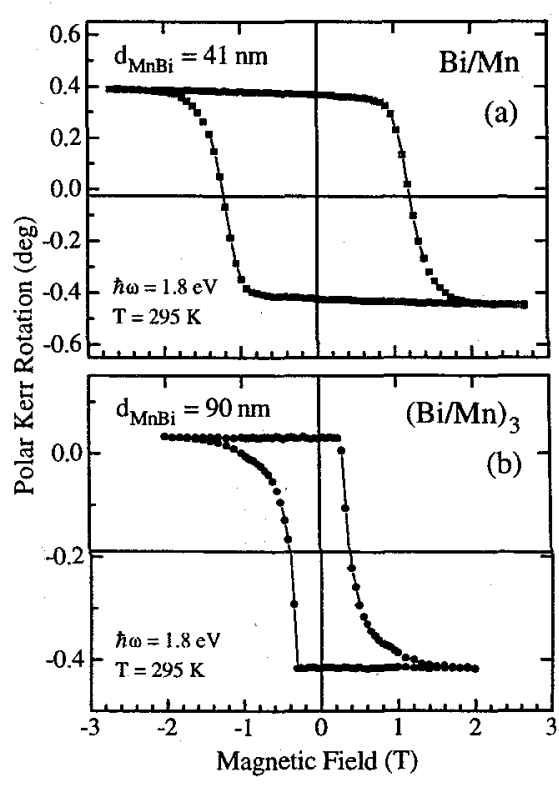

FIG. 5. Polar Kerr-hysteresis loops at a photon energy of $1.8 \mathrm{eV}$ of (a) a $\mathrm{Bi}(22 \mathrm{~nm}) / \mathrm{Mn}(19 \mathrm{~nm})$ bilayer and (b) a $(\mathrm{Bi} / \mathrm{Mn})_{3}$ sequence with a $\mathrm{Bi}$ and $\mathrm{Mn}$ layer thickness of 18 and $12 \mathrm{~nm}$, respectively. The loops are taken from the film side after annealing.

includes dipole interaction, wall energy, Zeeman energy, and an energy barrier describing the reversal of a single grain [12]. The film is assumed to consist of cells with height, $h$, and lateral dimension, $L$, on a square lattice. Using experimental values for $h$ and $L$, the model accurately accounts for the thickness dependence of the coercivity as demonstrated in Fig. 6 . The value of $h$ corresponds to the experimental film thickness while $L$ is estimated from the SEM pictures (Fig. 4). However, the coercivity is a factor of

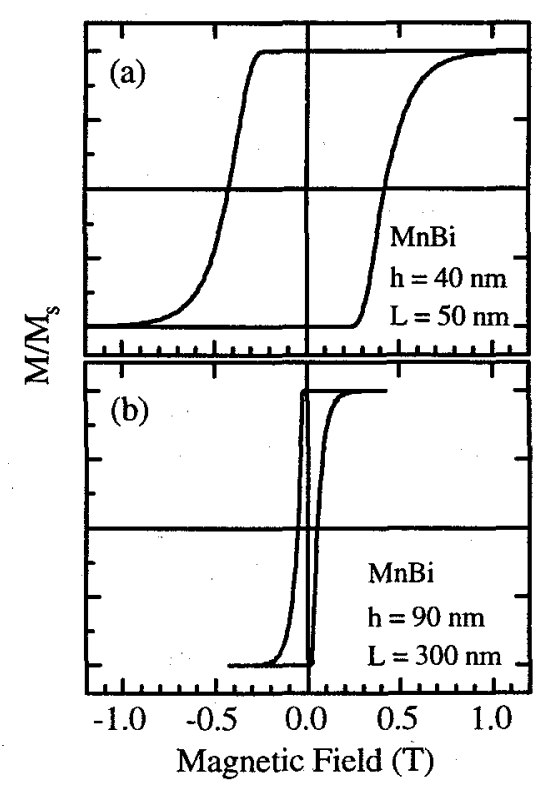

FIG. 6. Simulated hysteresis loops for a square lattice cell size of (a) height $h=40 \mathrm{~nm}$ and lateral dimension $L=50 \mathrm{~nm}$ and (b) $h=$ $90 \mathrm{~nm}$ and $L=300$ corresponding to a $\mathrm{MnBi}$ single grain. 


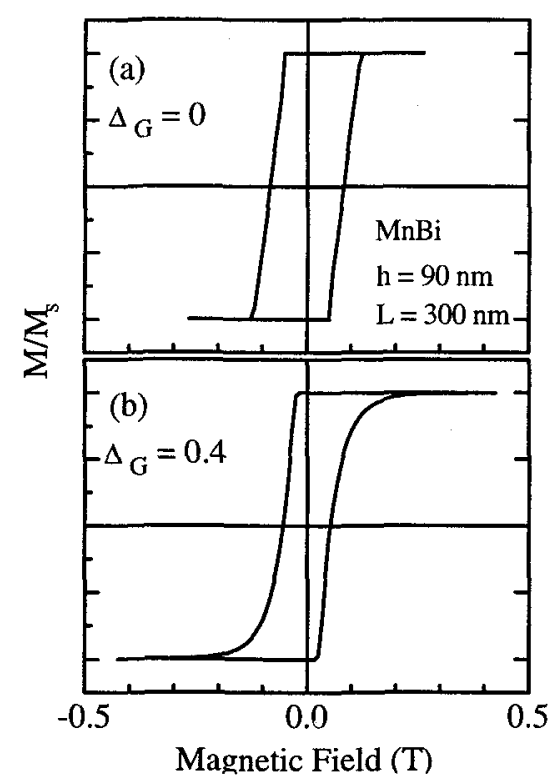

FIG. 7. Disorder dependence of the hysteresis loops for $h=90 \mathrm{~nm}$ and $L=300 \mathrm{~nm}$ for (a) $\Delta_{\mathrm{G}}=0$ (no disorder) and (b) $\Delta_{\mathrm{G}}=0.4$.

two smaller than the experimental value indicating that the model is too simple to predict correct values of $H_{\mathrm{c}}$.

The model can in addition explain the change in the magnetization reversal. As is evident from the SEM pictures, the $\mathrm{MnBi}$ grain size is randomly distributed and therefore, disorder should be incorporated into the model. This would yield a random distribution of $L$. As this is very difficult to simulate, due to the complicated evaluation of the dipole term, disorder is added to the model by assuming a random fluctuation of $L$ only in the Zeeman term, since this is the leading term being quadratic in $L$ [12]. The resulting hysteresis loop is plotted in Fig. 7 for a Gaussian distribution of $L$ of width of $\Delta_{\mathrm{G}}=0.4$ and compared to the case without disorder.

Adding a thin $\mathrm{Al}$ or $\mathrm{Pt}$ interlayer between two $\mathrm{Bi} / \mathrm{Mn}$ bilayers strongly reduces surface roughness as evidenced in the SEM pictures in Fig. 8. Comparing with single and double $\mathrm{Bi} / \mathrm{Mn}$ bilayers (see Fig. 4), surface roughness decreases to the value of a single $\mathrm{Bi} / \mathrm{Mn}$ bilayer. As demonstrated in an AFM study [9], the Pt produces a plateaulike structure when growing on a $\mathrm{Bi} / \mathrm{Mn}$ bilayer, explaining the smoothing of the surface.

As shown in Fig. 9, the corresponding polar Kerr loops, measured from the film side, display a different behavior for $\mathrm{Al}$ and $\mathrm{Pt}$ interlayers. When adding an $\mathrm{Al}$ interlayer of $d_{\mathrm{Al}} \geq 1.5 \mathrm{~nm}$, the Kerr loops develop a peculiar form. Starting at the highest positive magnetic field, magnetization reversal takes place in two steps. Shortly after reversing the field, at $H \cong-0.2 \mathrm{~T}$, the Kerr rotation, $\theta_{\mathrm{K}}$, shows a sharp decline before increasing again to a positive saturation value at $H \cong-1 \mathrm{~T}$. This behavior can be explained by assuming two decoupled magnetic layers with different values of $H_{\mathfrak{c}}$ and opposite sign of $\theta_{\mathrm{K}}$. The inset in Fig. 9(a) shows a superposition of two model loops with the proposed characteristics correctly describing the experimental data. An antiferromagnetic coupling between the two layers can be
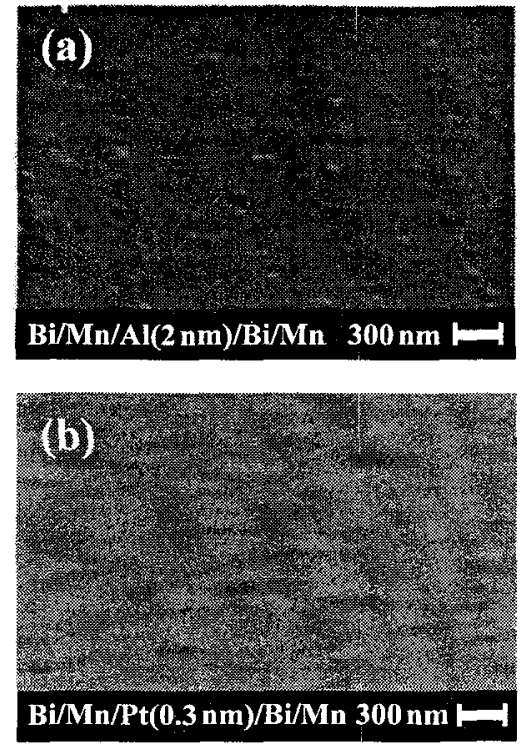

FIG. 8. SEM picture of (a) $\mathrm{Bi} / \mathrm{Mn} / \mathrm{Al}(2 \mathrm{~nm}) / \mathrm{Bi} / \mathrm{Mn}$ and (b) $\mathrm{Bi} / \mathrm{Mn} / \mathrm{Pt}(0.3 \mathrm{~nm}) / \mathrm{Bi} / \mathrm{Mn}$ with a $\mathrm{Bi}$ and $\mathrm{Mn}$ layer thickness of 18 and $12 \mathrm{~nm}$, respectively. Both pictures are taken before annealing. The characteristics do not change after annealing.

excluded as in that case the switching should take place before reversing the external field [8]. Measuring the Kerr hysteresis loops through the substrate [8], reveals an common square loop with negative $\theta_{\mathrm{K}}$ and a coercive field $H_{\mathrm{c}}=0.4 \mathrm{~T}$. In conclusion, by adding an $\mathrm{Al}$ interlayer with $d_{\mathrm{Al}} \geq 1.5 \mathrm{~nm}$ the $\mathrm{MnBi}$ film is divided into two decoupled magnetic layers, where the top one has a large $H_{\mathrm{c}}$ of $\equiv 1 \mathrm{~T}$, similar to a single $\mathrm{Bi} / \mathrm{Mn}$ bilayer, and the bottom one has a very small $H_{\mathrm{c}}$ of $\cong 0.4 \mathrm{~T}$, as in a thick $\mathrm{MnBi}$ film. Note that the bottom layer now manifests the same value of $H_{\mathrm{c}}$ as reported in samples

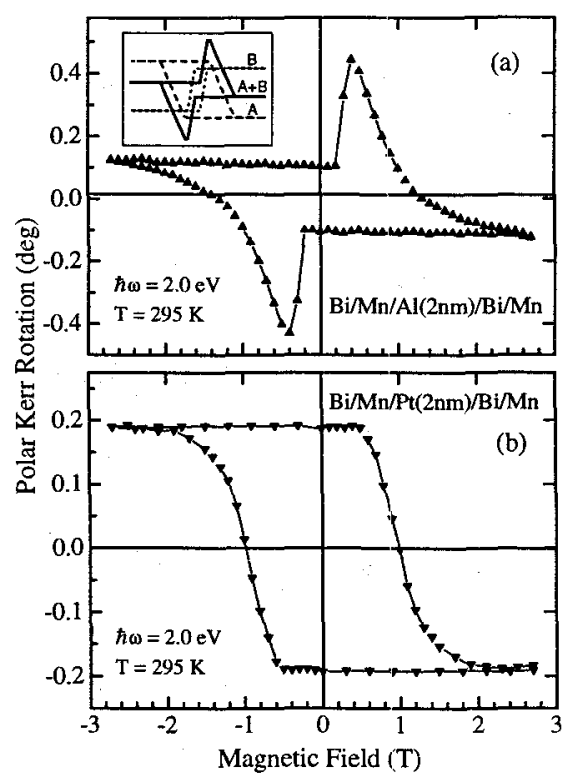

FIG. 9. Polar Kerr-hysteresis loops at a photon energy of $2.0 \mathrm{eV}$ of (a) $\mathrm{Bi} / \mathrm{Mn} / \mathrm{Al}(2 \mathrm{~nm}) / \mathrm{Bi} / \mathrm{Mn}$ and (b) $\mathrm{Bi} / \mathrm{Mn} / \mathrm{Pt}(2 \mathrm{~nm}) / \mathrm{Bi} / \mathrm{Mn}$. The loops are taken after annealing from the fillm side. 
with a protective $\mathrm{SiO}_{x}$ layer $[3,11]$.

Adding a Pt interlayer (see Fig. 9(b)), yields a square loop with $H_{\mathrm{c}}$ slightly reduced as compared to a single $\mathrm{Bi} / \mathrm{Mn}$ bilayer. By increasing the $\mathrm{Pt}$-interlayer thickness gradually from 0.3 to $2 \mathrm{~nm}, H_{\mathrm{c}}$ increases progressively from the value of a double $\mathrm{Bi} / \mathrm{Mn}$ bilayer to the value of a single $\mathrm{Bi} / \mathrm{Mn}$ bilayer [9]. This is surprising as the RBS data (see Fig. 3) show that the Pt interlayer merges completely with the $\mathrm{Bi} / \mathrm{Mn}$ bilayers. Hence, one would expect that the coercivity remains constant at the value of a double $\mathrm{Bi} / \mathrm{Mn}$ bilayer.

In order to understand the effect of adding $\mathrm{Al}$ and $\mathrm{Pt}$ interlayers or a protective $\mathrm{SiO}_{x}$ layer, let us discuss some consequences of the layering-deposition method. Deposition of a $\mathrm{Bi} / \mathrm{Mn}$ bilayer results in an average $\mathrm{Mn}_{x} \mathrm{Bi}_{1-x}$ composition where the $\mathrm{Mn}$ concentration $x_{\mathrm{Mn}}$ is obtained by

$$
x_{M n}=\frac{\rho_{M n} d_{M n}}{M_{M n}}\left(\frac{\rho_{M i n} d_{M n}}{M_{M n}}+\frac{\rho_{B i} d_{B i}}{M_{B i}}\right)^{-1} .
$$

Inserting $\rho_{\mathrm{Bi}}=9.8 \mathrm{~g} / \mathrm{cm}^{3}, \rho_{\mathrm{Mn}}=7.43 \mathrm{~g} / \mathrm{cm}^{3}, d_{\mathrm{Bi}}=18 \mathrm{~nm}$, $d_{\mathrm{Mn}}=12 \mathrm{~nm}, M_{\mathrm{Bi}}=209 \mathrm{~g} / \mathrm{mol}$ and $M_{\mathrm{Mn}}=55 \mathrm{~g} / \mathrm{mol}$, we get $x_{\mathrm{Mn}}=0.66$. This is far away from the ideal stoichiometry of $x_{\mathrm{Mn}}=0.5$. As evidenced by energy-dispersive $\mathrm{x}$-ray analysis, about $10 \%$ of the $\mathrm{Mn}$ evaporates during annealing. Furthermore, a small amount of $\mathrm{Mn}$ is bound at the surface by oxygen (see Fig. 3). As a result, the complete Bi layer but only part of the $\mathrm{Mn}$ layer is engaged in the $\mathrm{MnBi}$ formation process. We can calculate the average volume, $v_{\mathrm{Bi} / \mathrm{Mn}}$, per formula unit $\mathrm{MnBi}$ assuming a $\mathrm{Bi}$ and $\mathrm{Mn}$ layer thickness corresponding to the ideal stoichiometry

$$
v_{\mathrm{Bi} / \mathrm{Mn}}=\mathrm{v}_{\mathrm{Bi}}+\mathrm{v}_{\mathrm{Mn}}=\frac{1}{N_{\mathrm{A}}}\left(\frac{\mathrm{M}_{\mathrm{Bi}}}{\rho_{\mathrm{Bi}}}+\frac{M_{\mathrm{Mn}}}{\rho_{\mathrm{Mn}}}\right),
$$

where $N_{\mathrm{A}}$ is Avogadro's number. Inserting the values, we calculate $v_{\text {Bimn }}=47.7 \AA^{3}$. This has to be compared with the cell volume per formula unit of NiAs-type $\mathrm{MnBi}$, $v_{\mathrm{MnBi}}=\sqrt{3} / 4 a_{\mathrm{MnBi}}^{2} c_{\mathrm{MnBi}}=48.8 \AA^{3}$ using $a_{\mathrm{MnBi}}=4.29 \AA$ and $c_{\mathrm{MnBi}}=6.126 \AA[13,14]$. This is $2.3 \%$ larger than $v_{\mathrm{BiMn}}$. As a consequence, the film has to expand during crystallization generating internal pressure. This would lead to an increasing surface roughness when enlarging film thickness. The thicker the films the larger the disorder which is induced by the nucleation of individual crystallites. However, as has been discussed before, surface roughness develops already during deposition creating void space. Therefore, part of the internal stress induced by the volume increase during crystallization will be relaxed through the void space.

The change in coercivity behavior of $\mathrm{MnBi}$ films can also be explained by a volume increase. Depositing a hard interlayer, like $\mathrm{Al}_{2} \mathrm{O}_{3}$, or a protective layer, like $\mathrm{SiO}_{x}$, will restrict expansion of the crystallites perpendicular to the surface, leading to a pronounced lateral growth of the $\mathrm{MnBi}$ crystallites. This will lead to larger lateral dimensions and a better interconnection of individual grains reducing the wall energy at the grain boundary. Both effects reduce coercivity which explains the small values observed.

\section{MAGNETO-OPTIC PROPERTIES}

In the last section we will discuss the magneto-optic properties of $\mathrm{Al}$ and $\mathrm{Pt}$ doped $\mathrm{MnBi}$ thin films. The complex polar Kerr spectra in the remanence state and the reflectivity relative to an $\mathrm{Al}$ reference mirror of a $\operatorname{MnBiAl}(0.3 \mathrm{~nm})$ and two $\mathrm{MnBiPt}\left(d_{\mathrm{Pt}}\right)$ films, with $d_{\mathrm{Pt}}=0.3$ and $1.5 \mathrm{~nm}$, are shown in Fig. 10, as measured through the substrate. The remanence state is chosen because in that case no spurious effects of the Faraday rotation of the substrate are present that could falsify the spectra, especially in the UV part of the photon-energy range. Each spectra is divided by a factor of 1.5 to account for the interface-enhancement effect when measuring through the quartz substrate [15].

For the $\mathrm{MnBiAl}$ multilayer, two major peaks are observed in $\theta_{\mathrm{K}}$ at 1.9 and $3.4 \mathrm{eV}$ with an absorptive line shape. The maximum Kerr rotation amounts for both structures to $-0.9^{\circ}$, the latter being very advantageous for blue recording. The corresponding features in the Kerr ellipticity, $\eta_{K}$, exhibit dispersive line shapes. The maximum value, $\eta_{\mathrm{K}}=-0.9^{\circ}$, is found at $4.5 \mathrm{eV}$. The spectra are similar in size and shape for pure $\mathrm{MnBi}$ (not shown). Our measurements are in good agreement with recently published spectra $[1,16]$. However, these studies were made on $\mathrm{SiO}_{x}$-protected $\mathrm{MnBi}$ films. There, the peaks positions are also found at 1.9 and $3.4 \mathrm{eV}$ but, unlike our results, the second peak $\left(\theta_{K}=1.5^{\circ}\right)$ is smaller by $15 \%$ than the one at lower energy $\left(\theta_{K}=1.75^{\circ}\right)$. To compare with our findings, the rotations have to be corrected for the interface effect. This yields $\theta_{\mathrm{K}}=1.16^{\circ}$ and $1^{\circ}$ in fair agreement with our data.

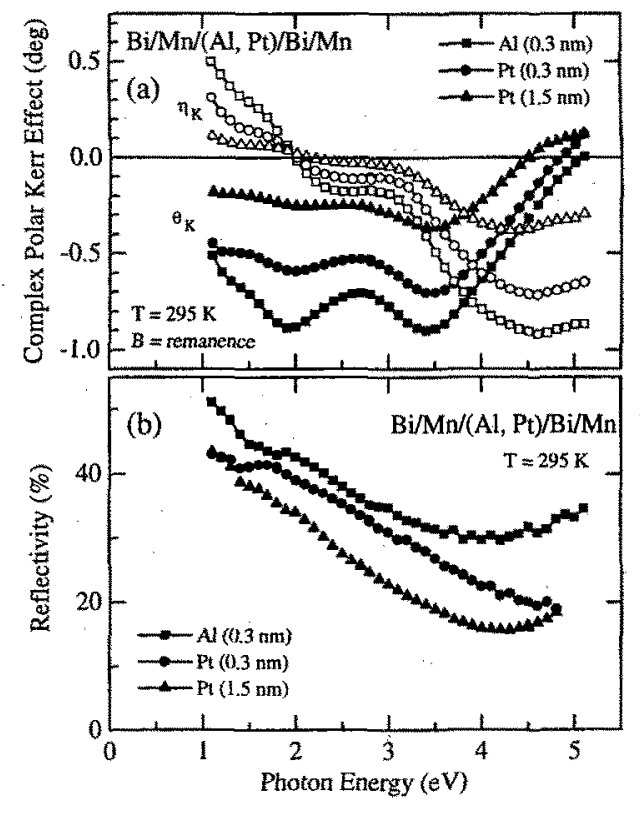

FIG. 10. (a) Complex polar Kerr spectra in the remanence state and (b) reflectivity of $\operatorname{MnBiAl}(0.3 \mathrm{~nm})(\mathbf{\omega}), \operatorname{MnBiPt}(0.3 \mathrm{~nm})(\bullet)$, and MnBiPt(1.5 nm) (A). Open symbols represent the Kerr ellipticity. Measurements were obtained through the substrate at room temperature. 
Adding $\mathrm{Pt}$ to $\mathrm{MnBi}$ reduces the Kerr rotation evenly across the photon-energy range except for the sample with a $2 \mathrm{~nm}$ thick Pt interlayer. Here, the first peak at $1.9 \mathrm{eV}$ is more reduced than the peak at $3.4 \mathrm{eV}$. In the same way, the magnetization, $\mu$, decreases as a function of Pt content from $\mu=536 \mathrm{kA} / \mathrm{m}$ to $304 \mathrm{kA} / \mathrm{m}$ for $d_{\mathrm{Pt}}=0.3$ and $1.5 \mathrm{~nm}$. Normalizing the maximum Kerr rotation, $\theta_{\mathrm{K}}^{\max }$ at $3.4 \mathrm{eV}$ to the magnetization, we get a ratio $\theta_{\mathrm{K}}^{\max } / \mu=1.31 \times 10^{-3}$ and $1.20 \times 10^{-3} \mathrm{deg} \mathrm{m} / \mathrm{kA}$, respectively, which is equal to within $10 \%$. This indicates that the addition of Pt reduces primarily the magnetization of the $\mathrm{MnBi}$ films. As a consequence, $\theta_{\mathrm{K}}$ is decreasing in the same way being in first order proportional to $\mu$. At present, we do not know what causes the magnetization reduction.

The reflectivity, $R$, as plotted in Fig. 10(b), decreases continuously with photon energy in all samples. It is the largest for the $\mathrm{Al}$ doped film where $R$ declines from $50 \%$ at $1.1 \mathrm{eV}$ to $30 \%$ at $4 \mathrm{eV}$. This is in agreement with published data [16]. The Pt-doped films have lower values of $R$ starting at $45 \%$ at $1.1 \mathrm{eV}$ and decreasing to $20 \%$ at $4 \mathrm{eV}$. Note that this is not an absolute measurement. Therefore, the reflectivity might have an absolute error of several percent but the relative changes should be real. If we calculate the shot-noise limited figure of merit, $F O M=\sqrt{R}\left(\theta_{\mathrm{K}}^{2}+\eta_{\mathrm{K}}^{2}\right)^{4 / 2}$, a value of $F O M=0.49^{\circ}$ is derived at $3 \mathrm{eV}$. This is considerably more than in $\mathrm{Co} / \mathrm{Pt}$ multilayers [17] explaining the high interest in this compound as a potential magnetooptic storage material.

As $a b$-initio calculations of the Kerr effect have made an immense progress in the last few years, we would like to compare our data with recent first-principles studies on the Kerr effect of MnBi [18, 19]. The theoretical studies show that the high intrinsic Kerr effect in $\mathrm{MnBi}$ is due to the coincidence of three favorable issues: (i) a large magnetic moment of $3.7 \mu_{\mathrm{B}}$, (ii) a large spin-orbit coupling of $\mathrm{Bi}$, and (iii) a large hybridization between the $d$ and $p$ states of $\mathrm{Mn}$ and $\mathrm{Bi}$. This leads to strong, dipole-allowed $\mathrm{Mn}-3 d \rightarrow \mathrm{Bi}-5 p$ as well as $\mathrm{Bi}-5 p \rightarrow \mathrm{Mn}-4 d$ transitions which are magnetooptically active because of (i) and (ii). Although the first peak at $1.9 \mathrm{eV}$ is computed in good agreement with experiment [18] the second peak at $3.4 \mathrm{eV}$ does not appear in the primary calculations. At present, there are two approaches to account for the high-energy peak. One is by assuming a change in stoichiometry from $\mathrm{MnBi}$ to a Heusler-like $\mathrm{Mn}_{2} \mathrm{Bi}$ [18], the other one is by assuming oxygen filling in the interstitial sites of the MnBi unit cell [19]. Future measurements have to clarify if any of these mechanisms is responsible for the peak at $3.4 \mathrm{eV}$ or if the primary calculations need to be modified.

\section{CONCLUSIONS}

Regarding the suitability of $\mathrm{MnBi}$ films as a magnetooptic recording material the following conclusions can be made. $\mathrm{MnBi}$ has a very high room-temperature Kerr rotation $\theta_{\mathrm{K}}$ in the blue spectral range reaching $1^{\circ}$. By using a sequential deposition technique, $\mathrm{Al}$ and $\mathrm{Pt}$ interlayers can be added without degrading $\theta_{\mathrm{K}}$ considerably. The interlayers improve surface quality, reduce grain size and can be used to optimize the coercivity $\mathrm{H}_{\mathrm{c}}$. The large grain size and surface roughness is found to be due to the growth behavior of $\mathrm{Mn}$ on $\mathrm{Bi}$ during deposition. Consequently, co-evaporation of $\mathrm{Mn}$ and $\mathrm{Bi}$ might be the key to an efficient reduction of grain size and surface roughness. Concerning the major hindrances for an application, further magneto-optic measurements at elevated temperatures will be performed in near future to investigate the influence of the $\mathrm{Al}$ and $\mathrm{Pt}$ interlayers on the structural phase transition and the $\mathrm{H}_{c}$ anomaly.

\section{ACKNOWLEDGMENT}

This work has been financially supported by the German Ministry of Education, Science, Research and Technology, BMBF, under grant no. FKZ 13 N 6178/2.

[1] G. Q. Di, S. Iwata, S. Tsunashima, and S. Uchiyama, J. Magn. Magn. Mater. 104-107, 1023 (1992).

[2] T. Chen and W. E. Stutius, IEEE Trans. Magn. 10, 581 (1974).

[3] X. Guo, X. Chen, Z. Altounian, and J. O. Ström-Olsen, Phys. Rev. B 46, 14578 (1992).

[4] U. Rüdiger, H. Berndt, A. Schirmeisen, P. Fumagalli, and G. Güntherodt, J. Appl. Phys. 78, 5391 (1995).

[5] U. Rüdiger, P. Fumagalli, P. Dworak, A. Schirmeisen, and G. Güntherodt, J. Appl. Phys. 79, 6203 (1996).

[6] J. X. Shen, R. D. Kirby, D. J. Sellmyer, and Y. J. Wang, J. Appl. Phys. 69, 5984 (1991).

[7] A. Morisako and M. Matsumoto, J. Appl. Phys. 61, 4281 (1987).

[8] U. Rüdiger, P. Fumagalli, H. Berndt, A. Schirmeisen, G. Güntherodt, and B. Holländer, J. Appl. Phys. (1996), in press.

[9] U. Rüdiger, P. Fumagalli, P. Dworak, and G. Güntherodt, IEEE Trans. Magn. (1996), to be published.

[10] C. Kittel, Phys. Rev. 73, 810 (1948).

[11] D. Chen, J. F. Ready, and E. Bernal, J. Appl. Phys. 39, 3916 (1968).

[12] U. Nowak, U. Rüdiger, P. Fumagalli, and G. Güntherodt, Phys. Rev. B, to be published.

[13] A. F. Andresen, W. Hälg, P. Fisher, and E. Stoll, Acta Chem. Scand. 21, 1543 (1967),

[14] X. Guo, Z. Altounian, and J. O. Ström-Olsen, J. Appl. Phys. 69, 6067 (1991).

[15] P. Fumagalli, T. S. Plaskett, T. R. McGuire, R. J. Gambino, and N. A. Bojarczuk, Phys. Rev. B 46, 6187 (1992).

[16] G. Q. Di and S. Uchiyama, Phys. Rev. B 53, 3327 (1996).

[17] H. Brändle, D. Weller, J. C. Scott, S. S. P. Parkin, and C.-J. Lin, IEEE Trans. Magn. 28, 2967 (1992).

[18] P. M. Oppeneer, V. N. Antonov, T, Kraft, E. Eschrig, A. N. Yaresko, and A. Y. Perlov, Phys. Rev B, to be published.

[19] J. Köhler and J. Kübler, Annual Meeting of the German Physical Society, Regensburg, March 25-29, 1996. 